\title{
Rayleigh-Taylor Instabilities in Young Supernova Remnants Undergoing Efficient Particle Acceleration
}

\author{
John M. Blondin and Donald C. Ellison \\ Department of Physics, North Carolina State University, Raleigh, NC 27695-8202 \\ John_Blondin@ncsu.edu, Don_Ellison@ncsu.edu
}

\begin{abstract}
We employ hydrodynamic simulations to study the effects of high shock compression ratios, as expected for fast shocks with efficient particle acceleration, on the convective instability of driven waves in supernova remnants. We find that the instability itself does not depend significantly on the compression ratio, $\sigma$, with the growth rates and the width of the mixing region at saturation being comparable for the range of ratios we studied; $4 \leq \sigma \leq 21$. However, because the width of the interaction region between the forward and reverse shocks can shrink significantly with increasing $\sigma$, we find that convective instabilities can reach all the way to the forward shock front if compression ratios are high enough. Thus, if supernova blast waves accelerate particles efficiently, we expect the forward shock to be perturbed with small amplitude, small wavelength bumps, and to find clumps and filaments of dense ejecta material in the vicinity of the shock. In addition and in contrast to situations where $\sigma \leq 4$, any enhancement of the radial magnetic field from Rayleigh-Taylor instabilities will also extend all the way to the shock front and this may help explain the slight dominance of radial fields long seen in polarization measurements of young remnants like Tycho.
\end{abstract}

Subject headings: hydrodynamics - instabilities - shock waves - ISM: supernova remnants: cosmic rays

\section{INTRODUCTION}

The presence of convective or Rayleigh-Taylor (R-T) instabilities in young, ejecta-dominated supernova remnants (SNRs) has been argued for a long time (e.g., Gull 1973; Shirkey 1978; Dickel et al. 1989) and examined in detail with numerical simulations of idealized models (e.g., Chevalier et al. 1992; Jun \& Norman 1996a; Wang \& Chevalier 2000). These instabilities may be important for mixing ejecta out to, and possibly past, the forward shock, and for modifying the morphology of an otherwise spherical outer blast wave and/or reverse shock. One of the best studied young SNRs, Cas A exhibits ejecta material out to and beyond the nominal radius of the forward shock, as evidenced by optical knots of enriched gas (Fesen \& Gunderson 1996), as well as spectral analysis of X-ray knots and filaments (Hughes et al. 2000a). The outer blastwave of Cas A, as seen in X-ray emission, is roughly spherical, but possesses many bumps and protrusions that may be the result of the convective instability. A more extreme case of an irregular blastwave is 1986J. VLBI observations of this SNR (Bartel et al. 1991) show a shell of radio emission with large radial protrusions, suggesting that very young SNRs can exhibit large deviations from spherical symmetry.

Another characteristic of young SNRs that may originate with the convective instability associated with the contact discontinuity is the slight dominance of a radial magnetic field as deduced from radio polarization measurements (Dickel et al. 1991). Jun \& Norman (1996b) suggested that the polarization observations could be explained by convective instabilities dragging out an ambient magnetic field as the R-T fingers pushed from the contact discontinuity out toward the forward shock.

Despite the belief that convective instabilities 
alone should play an important role in perturbing the forward shock and generating a strong magnetic field, attempts to demonstrate this have failed. Chevalier et al. (1992) used a linear perturbation analysis and two-dimensional hydrodynamic simulations to study this instability, and found that the mixing region did not reach the forward shock. This result was confirmed by Jun \& Norman (1996a) and found to hold true even in the presence of rapid cooling of the shocked ejecta (Chevalier \& Blondin 1995). Jun \& Norman (1996b) showed that this limited mixing region was inconsistent with observations, for it produced a SNR with a two-shell structure when viewed in radio-synchrotron emission: an inner shell of strong emission and a dominant radial magnetic field, and an outer shell of weaker emission with a dominant tangential field. However, Jun, Jones, \& Norman (1996) found that including small cloudlets in the surrounding circumstellar medium (CSM) could enhance the growth of the Rayleigh-Taylor fingers enough so they reached all the way to the shock front.

All of this previous work concerning R-T instabilities has assumed the supernova ejecta and CSM can be treated as an ideal gas, typically modeled with a ratio of specific heats, $\gamma=5 / 3 .{ }^{1}$ However, these assumptions may not be appropriate for young SNRs, where the strong shock waves are expected to be efficient accelerators of energetic particles (i.e., cosmic rays). ${ }^{2}$ It has long been believed that SNRs are the primary sources of galactic cosmic rays below $\sim 10^{15} \mathrm{eV}$ (e.g., Axford

\footnotetext{
${ }^{1}$ For discussions of other types of instabilities in shocks accelerating cosmic rays see, for example, Drury \& Falle (1986) and McClements et al. (1996).

${ }^{2}$ In the heliosphere, where shocks are observed directly with spacecraft, there is clear evidence that the quasi-parallel earth bow shock (with a sonic Mach number, $M_{\mathrm{S} 0}<10$ ) can place $10-30 \%$ of the solar wind kinetic energy flux into superthermal particles (e.g., Ellison et al. 1990). Quasiperpendicular interplanetary shocks (IPSs) also accelerate ambient particles but generally with lower efficiencies due to their lower Mach numbers (generally $M_{\mathrm{S} 0} \lesssim 3$ for IPSs) (e.g., Baring et al. 1997). In a few cases, however, IPSs with higher than average Mach numbers have been observed to accelerate particles with efficiencies comparable to the quasi-parallel Earth bow shock (Eichler 1981; Terasawa et al. 1999). Hybrid plasma simulations showing direct injection and acceleration of ambient particles are reasonably consistent with these observations (e.g., Scholer et al. 1992), as are convection-diffusion models (e.g., Kang \& Jones 1997).
}

1981). However, for this to be the case, the acceleration mechanism must be efficient and place on the order of $10 \%$ or more of the total ejecta kinetic energy into relativistic particles (e.g., Blandford \& Eichler 1987; Drury, Markiewicz, \& Völk 1989; Dorfi 2000). Radio observations have long been proof that SNRs produce $\mathrm{GeV}$ electrons and recent evidence of X-ray synchrotron emission in young SNRs suggests they can produce $\mathrm{TeV}$ electrons as well (e.g., Koyama et al. 1995; Mastichiadis \& de Jager 1996; Allen et al. 1997; Tanimori et al. 1998; Reynolds \& Keohane 1999). While direct proof of ion acceleration in SNRs remains elusive, there is a growing body of evidence linking the efficient production of cosmic-ray ions by the forward and reverse shocks with the thermal properties of the shock-heated X-ray emitting gas (e.g., Dorfi \& Böhringer 1993; Decourchelle, Ellison, \& Ballet 2000; Hughes, Rakowski, \& Decourchelle 2000b).

One of the important structural effects of efficient particle acceleration is a shock compression ratio greater than 4 (e.g., Ellison \& Eichler 1984; Berezhko \& Ellison 1999). For an ideal gas in the absence of particle acceleration, a high Mach number shock produces a compression ratio,

$$
\sigma \simeq \frac{\gamma+1}{\gamma-1}
$$

For the nominal value of $\gamma=5 / 3, \sigma=4$. However, when efficient particle acceleration occurs, relativistic particles can be created and substantially contribute to the pressure, and superthermal particles can escape from the shock. These two effects combine to produce a shocked plasma which acts to a large extent like a gas with an effective ratio of specific heats, $\gamma_{\mathrm{eff}}<5 / 3$, allowing arbitrarily large compression ratios. In fact, it has been shown (Berezhko \& Ellison 1999) that nonlinear, diffusive shock acceleration yields

$$
\sigma \simeq\left\{\begin{array}{lll}
1.3 M_{\mathrm{S} 0}^{3 / 4} & \text { if } & M_{\mathrm{S} 0}^{2}>M_{\mathrm{A} 0} \\
1.5 M_{A 0}^{3 / 8} & \text { if } & M_{\mathrm{S} 0}^{2}<M_{\mathrm{A} 0}
\end{array} .\right.
$$

Here, $M_{\mathrm{S} 0}\left(M_{\mathrm{A} 0}\right)$ is the upstream sonic (Alfvén) Mach number (see also Kazanas \& Ellison 1986; Malkov 1997). Furthermore, the increase in compression ratio is accompanied by a decrease in the post-shock temperature which links the efficient production of superthermal particles with the thermal properties of the shock-heated, X-ray emitting gas. 
Of particular importance for the work reported here, the increased compression ratio also implies that the region between the forward and reverse shocks in young SNRs will be significantly narrower and denser when acceleration is efficient compared to the case where little energy is placed in relativistic particles. The increased density and narrower spatial extent of the interaction region leads to large density gradients suggesting that R$\mathrm{T}$ instabilities may have faster growth rates and larger amplitudes at saturation than is commonly assumed (Decourchelle, Ellison, \& Ballet 2000).

In this paper we explore the possibility that the familiar convective instability in young SNRs may be altered by high compression ratios. We use a well-tested hydrodynamic simulation of evolving SNRs, only replacing the standard $\gamma=5 / 3$ with an effective ratio of specific heats, $\gamma_{\text {eff }}<5 / 3$. A softer equation of state (a low $\gamma_{\text {eff }}$ ) causes the shocked plasma to be more compressible, yielding shock compression ratios $\sigma>4$, consistent with those expected when particle acceleration is efficient.

Somewhat unexpectedly, we find that the convective instability growth rate and mixing length are almost independent of the compression ratio. However, since the region between the forward and reverse shocks narrows as $\sigma$ increases, the likelihood that Rayleigh-Taylor fingers reach and perturb the forward shock is strongly correlated with $\sigma$ and, therefore, with the efficiency of cosmic-ray production. If $\sigma$ is large enough, the convective instabilities do reach and perturb the forward shock making it likely that clumps and filaments of dense ejecta material will be found there.

Since the magnetic field is also compressed, more perturbed shocks may be expected to show stronger radio emission and possibly more intense $\mathrm{TeV}$ emission from inverse-Compton and/or piondecay than unperturbed shocks. We have not, however, included magnetic fields in our hydrodynamic simulations.

In section 2 we discuss the impact of a low value of $\gamma_{\text {eff }}$ on a one-dimensional dynamical model of young SNRs. In section 3 we present hydrodynamic simulations in one, two, and three dimensions, and in section 4 we discuss the approximations and implications of our numerical results.

\section{A DYNAMICAL MODEL}

The dynamical evolution of young SNRs is determined by the interaction of the stellar ejecta, one to several solar masses of material thrown out by the SN explosion, with the surrounding circumstellar medium. This interaction is characterized by a two shock structure: a forward shock driven ahead of the ejecta into the CSM, and a reverse shock that decelerates the supersonically expanding ejecta in the vicinity of the interaction region. To model this ejecta-dominated evolution, we begin with the self-similar driven wave (SSDW) model (Chevalier 1982a), although we have found results similar to those reported here for the exponential ejecta model (Dwarkadas \& Chevalier 1998).

Chevalier (1982a) provided spherically symmetric, self-similar solutions to the structure of ejectadominated SNRs under the assumption that the mass density in both the ejecta and the CSM could be described by power laws. Since the steep power law density profiles for the ejecta lead to an infinite ejecta mass, we follow Chevalier and institute a cutoff in the ejecta density profile at small radii. Therefore, at radii less than some critical value, $r_{c}=v_{c} t$, the ejecta density is taken to be a spatially constant plateau, while beyond this radius it follows a power law, i.e.,

$$
\rho_{\mathrm{ej}}(r, t)= \begin{cases}\rho_{c} v_{c}^{n} r^{-n} t^{n-3} & \text { for } \quad r>v_{c} t \\ \rho_{c} t^{-3} & \text { for } r<v_{c} t\end{cases}
$$

where $t$ is time, the constant $\rho_{c}$ is given by

$$
\rho_{c}=\frac{5 n-25}{2 \pi n} E_{\mathrm{sn}} v_{c}^{-5},
$$

the velocity at the intersection of the ejecta density plateau and the power law is given by

$$
v_{c}=\left(\frac{10 n-50}{3 n-9} \frac{E_{\mathrm{sn}}}{M_{\mathrm{ej}}}\right)^{1 / 2}
$$

and $n$ is a constant index. These quantities, $\rho_{c}$ and $v_{c}$, are related to the constant $g$ in Chevalier (1982a) by $\rho_{c} v_{c}^{n}=g^{n}$. Here, $E_{\mathrm{sn}}$ and $M_{\mathrm{ej}}$ are the kinetic energy and mass of the ejecta, respectively, and it's clear that fixing the plateau allows us to relate the self-similar parameters to physical quantities normally associated with SNRs. Furthermore, we restrict our examples to times 
such that $r_{c}$ is less than the radius of the reverse shock to avoid complications from multiply reflected shocks etc., and to make direct comparisons between our numerical results and selfsimilar solutions straightforward.

The density of the ambient circumstellar medium is also described by a power law in radius in the Chevalier (1982a,b) solution, i.e.,

$$
\rho_{a}=q r^{-s}
$$

where $s=0$ corresponds to a uniform density (in which case $q$ is the ambient density) and $s=2$ represents the density profile of a steady-state stellar wind from the supernova progenitor of speed, $v_{w}$, and mass loss rate, $d M / d t$, such that $q=$ $(d M / d t) /\left(4 \pi v_{w}\right)$.

Thus, provided that the edge of the ejecta plateau region has not yet reached the reverse shock, the radius and expansion velocity of the forward shock are:

$$
\begin{aligned}
& R_{1}=b\left(\frac{\rho_{c} v_{c}^{n}}{q}\right)^{1 /(n-s)} t^{(n-3) /(n-s)} \\
& V_{1}=\left(\frac{n-3}{n-s}\right) \frac{R_{1}}{t}
\end{aligned}
$$

and the fluid variables in the shocked region immediately behind the forward shock are:

$$
\begin{aligned}
\rho_{1} & =\frac{\gamma_{\mathrm{eff}}+1}{\gamma_{\mathrm{eff}}-1} q R_{1}^{-s} \\
u_{1} & =\frac{2}{\gamma_{\mathrm{eff}}+1}\left(\frac{n-3}{n-s}\right) \frac{R_{1}}{t}, \\
p_{1} & =\frac{2 q}{\gamma_{\mathrm{eff}}+1}\left(\frac{n-3}{n-s}\right)^{2} \frac{R_{1}^{2-s}}{t^{2}}
\end{aligned}
$$

where $\rho$ is the density, $u$ is the fluid speed, $p$ is the pressure, and the subscript 1 indicates values at the forward shock. Note that all of the dependence on the ratio of specific heats in eq. (7) is contained in the constant $b$, which is related to parameters defined in Chevalier (1982a) through the expression $b=\left(R_{1} / R_{c}\right) A^{1 /(n-s)}$. The calculated values of the constant $A$ and the ratio of the forward shock radius to the radius of the contact discontinuity for $\gamma=5 / 3$ for various $n$ and $s$ are given in Table 1 of Chevalier (1982a).

We note that in an attempt to predict the $\gamma$-ray flux from pion-decay, Chevalier (1983) considered two-fluid, self-similar solutions where one fluid was a thermal gas with $\gamma=5 / 3$ and the other was a relativistic gas (i.e., cosmic rays) with $\gamma=4 / 3$. The effective ratio of specific heats was determined by

$$
\gamma_{\mathrm{eff}}=\frac{5+3 w}{3(1+w)},
$$

where $w$ was the (constant) fraction of total pressure made up by relativistic particles at the shock. Chevalier did not address the possible effects $\gamma_{\text {eff }}$ would have on R-T instabilities nor attempt to model the effects of particle escape during acceleration and assumed $4 / 3 \leq \gamma_{\text {eff }} \leq 5 / 3$ with a maximum $\sigma=7$ in his calculations. Chevalier did note, however, that cosmic rays diffusing from the shocked gas might produce a dense shell with $\sigma>7$ similar to that which occurs in the later radiative phase.

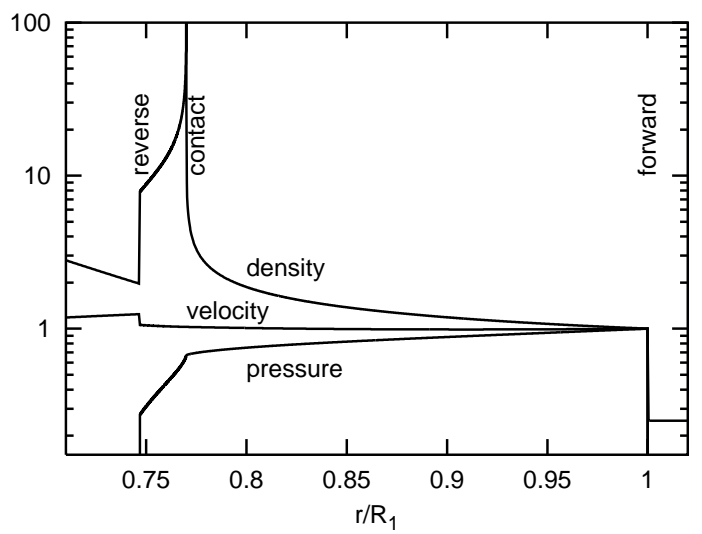

Fig. 1. - Internal structure of a SSDW with $n=7, s=2$, and $\gamma=5 / 3$ propagating to the right. The contact discontinuity separates the shocked ejecta on the left and the shocked CSM on the right. The radius is scaled to the position of the forward shock and the density, pressure, and velocity are scaled to their values just inside the forward shock.

The structure of a SSDW is illustrated in Figure 1, which shows the radial profiles of density, pressure, and velocity for values of $n=7$ and $s=2$. The interaction region is bounded by the forward and reverse shocks, which for this example have compression ratios $\sigma=4$ corresponding to a high Mach number adiabatic shock in an ideal fluid with $\gamma=5 / 3$. The pressure is monotonically increasing from the reverse shock to the forward 
shock, as all of the shocked gas is gradually decelerating.

An important characteristic of these solutions for $s=2$ is the convectively unstable region ahead of the contact discontinuity (Chevalier et al. 1992). This region is marked by opposing signs of the pressure and density gradients. The positive pressure gradient is gradually decelerating the shocked ejecta and shocked CSM, but the negative density gradient implies that the denser gas is being decelerated by lower density gas; a situation that is subject to the familiar R-T instability. A similar situation holds for $s=0$ solutions, but in this case the opposing gradients are behind the contact discontinuity rather than in front.

While the idealized self-similar hydrodynamical model has been very successful in describing general aspects of SNR evolution, it is clearly incomplete in some important ways. For instance, the hydrodynamic model ignores any effects from a magnetic field, the one-dimensional approximation eliminates the ability to describe irregularities in the ambient conditions or Rayleigh-Taylor generated instabilities, and particle acceleration at the collisionless forward and reverse shocks is neglected. Here we focus on how efficient cosmic-ray production influences R-T instabilities by setting $\gamma_{\text {eff }}<5 / 3$. We find that lowering $\gamma_{\text {eff }}$ does not alter the deceleration of the blast wave significantly (the radius of the forward shock still follows the self-similar result $\left.R_{1} \propto t^{(n-3) /(n-s)}\right)$, but it does shrink the width of the region between the forward and reverse shocks and alters the internal structure of the self-similar driven wave.

We list some of the parameters describing SSDWs for a range of $\gamma_{\text {eff }}$ in Table 1 and, for $\gamma=5 / 3$, these values are identical to those in Table 1 of Chevalier (1982a). These parameters, as well as the radial profiles shown in Figure 2, were generated following the procedure outlined in Chevalier (1982a). Note in particular that the value of $b$, which contains the $\gamma_{\text {eff }}$ dependence, changes by less than $30 \%$ as the compression ratio is varied from 4 to 21 . Thus the velocity of the blast wave is not strongly affected by the value of the compression ratio. In contrast, the width of the interaction region shrinks considerably as the compression ratio increases; between $\sigma=4$ and 21, the width shrinks by a factor of 4 in the case of $n=7$ and $s=0$.

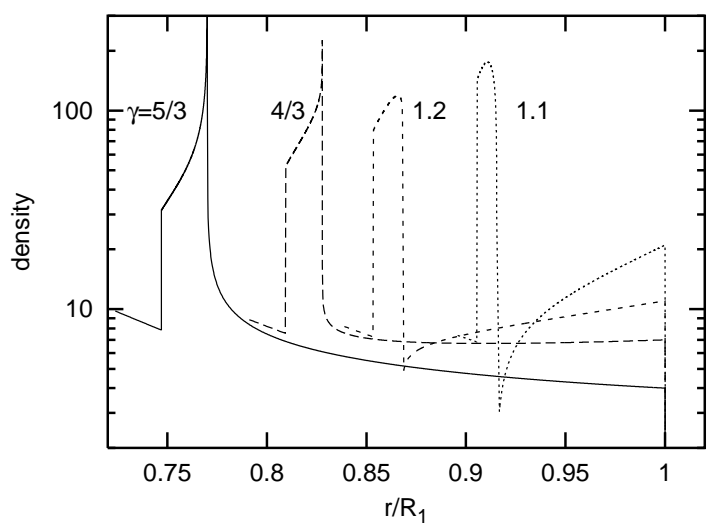

Fig. 2.- Radial profile of the gas density in SSDWs with $n=7$ and $s=2$ for values of $\gamma_{\mathrm{eff}}$ as labeled. Note the change in sign of the density gradient behind the forward shock. These results were obtained with the analytic solution of Chevalier (1982a), but essentially identical results are obtained from our one-dimensional hydrodynamic simulations. Here and in Figure 3, the density is scaled to its value immediately ahead of the forward shock.

The radial profiles of SSDWs for different values of $\gamma_{\text {eff }}$ are shown in Figure 2. While the increase in the compression ratio and decrease in the width of the interaction region are clearly evident, there is also a change in the density gradient of the shocked CSM. As gas moves from the forward shock to the contact discontinuity it compresses/expands adiabatically to match the relatively constant pressure of the interaction region. A lower $\gamma_{\text {eff leads to a }}$ slower drop in pressure due to radial expansion, so the gas does not need to be compressed as much as with a higher $\gamma_{\text {eff. }}$. The result is a more positive density gradient in the shocked CSM for a smaller $\gamma_{\text {eff. }}$ From Figure 2 we see that this can even lead to a change in the sign of the density gradient, which may in turn affect the stability properties of the waves. Nonetheless, we expect the low $\gamma_{\text {eff }}$ driven wave to remain unstable, as there is still a shell of dense shocked ejecta being decelerated by lower-density shocked CSM.

\section{HYDRODYNAMIC SIMULATIONS}

To examine the effects of different compression ratios on the convective stability of driven 
waves, we use the Virginia Hydrodynamics (VH1) time-dependent hydrodynamic numerical code in one, two, and three dimensions. All of the simulations were computed in spherical geometry on a numerical grid with 500 zones in each dimension. This resolution is comparable to the highest resolution simulations of Chevalier et al. (1992). Based on their results, we expect that a higher resolution simulation would show more small scale mixing, but the large-scale dynamics of the problem would not change. The angular extent of the simulation domain was chosen to produce roughly square numerical zones, i.e., $R \Delta \theta \approx \Delta R$. Periodic boundary conditions were applied in both the $\theta$ and $\phi$ directions for the multidimensional simulations. The radial boundary conditions were set to match the relevant power laws. The numerical grid was expanded to follow the forward blast wave so the evolution could be tracked for many expansion times.

We ran $1 \mathrm{D}$ and $2 \mathrm{D}$ simulations with the four combinations of $s=0,2$ and $n=7,12$. For each $(n, s)$ pair we ran four simulations with $\gamma_{\mathrm{eff}}=$ $5 / 3,4 / 3,1.2$, and 1.1 . In the following discussions we will focus on the set of simulations with $n=7$ and $s=2$ as a specific example, but the results are qualitatively the same for all of these parameter choices.

\subsection{D SIMULATIONS}

We ran 1D simulations for all the cases listed in Table 1 as a check on our ability to numerically recreate the analytic, self-similar solutions. The 1D hydrodynamic simulations were initialized with appropriate density power laws, and allowed to evolve until a self-similar state was reached. The gas pressure in the ejecta and CSM was kept sufficiently low to ensure that the Mach numbers of the forward and reverse shocks were always greater than 100 .

In Figure 3 we show the results of our most extreme case with $n=12$ and $s=0$ and $\gamma=1.1$ overlayed on the semi-analytic solution obtained by numerically integrating an ordinary differential equation (Chevalier 1982a). This particular SSDW is very thin and possesses steep gradients, making it a difficult test case for the hydrodynamic code. Nonetheless, our simulation does a reasonably good job of matching the analytic solution; note in particular the relative sharpness of

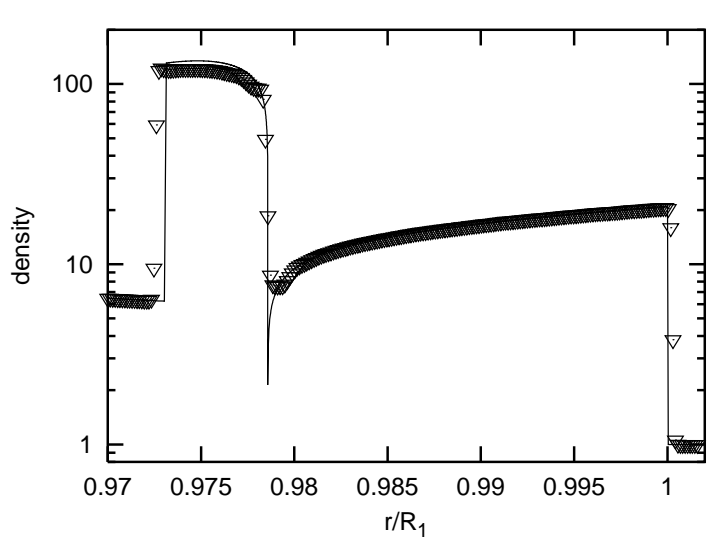

Fig. 3.- The density profile of a driven wave for the relatively extreme case with $n=12, s=0$, and $\gamma_{\text {eff }}=1.1$. The solid line is the self-similar analytic solution and the triangles are from the 1D hydrodynamic simulation.

the shock fronts and contact discontinuity. In the less extreme cases, the match between the simulation and the analytic solution is better than shown in Figure 3. However, the very sharp density peak at the contact discontinuity in the $s=2$ solutions, and the density trough in the $s=0$ solutions, are typically smoothed out over a few zones by this hydrodynamic method.

\subsection{D SIMULATIONS}

The multi-dimensional simulations were initiated with the analytic SSDW solutions normalized to $R_{1}=1, V_{1}=1$, and a preshock density of unity. The simulation thus begins at an initial time of $t=(n-3) /(n-s)$ in these normalized units. The initial solution is mapped onto a spherical grid with a radial span roughly twice the width of the interaction region. The convective instability was seeded by adding acoustic noise to the shocked interaction region. These simulations were evolved long enough for the convective instability to reach saturation, such that the mixing region is in a quasi-self-similar state with the growth of R-T fingers matched by their destruction due to shearing and advection. This typically required $\sim 6--8$ doubling times of the blast wave radius, and beyond this time, the results are qualitatively independent of the seed noise. 
Table 1: Numerically derived parameters describing spherically symmetric driven waves. The subscript $1(2)$ indicates values immediately behind the forward (reverse) shock.

\begin{tabular}{rrrcccrrr}
\hline \hline$s$ & $n$ & $\sigma$ & $\gamma$ & $R_{2} / R_{1}$ & $b$ & $\rho_{2} / \rho_{1}$ & $p_{2} / p_{1}$ & $u_{2} / u_{1}$ \\
\hline 0 & 7 & 4 & 1.67 & 0.792 & 1.212 & 1.34 & 0.47 & 1.253 \\
0 & 7 & 7 & 1.33 & 0.865 & 1.125 & 1.21 & 0.51 & 1.118 \\
0 & 7 & 11 & 1.20 & 0.907 & 1.081 & 1.14 & 0.53 & 1.066 \\
0 & 7 & 21 & 1.10 & 0.947 & 1.044 & 1.08 & 0.54 & 1.030 \\
\hline 2 & 7 & 4 & 1.67 & 0.747 & 0.996 & 7.8 & 0.27 & 1.058 \\
2 & 7 & 7 & 1.33 & 0.810 & 0.897 & 7.5 & 0.31 & 0.978 \\
2 & 7 & 11 & 1.20 & 0.853 & 0.840 & 7.2 & 0.33 & 0.960 \\
2 & 7 & 21 & 1.10 & 0.906 & 0.782 & 6.8 & 0.35 & 0.962 \\
\hline 0 & 12 & 4 & 1.67 & 0.869 & 0.977 & 7.2 & 0.60 & 1.255 \\
0 & 12 & 7 & 1.33 & 0.922 & 0.925 & 6.7 & 0.63 & 1.128 \\
0 & 12 & 11 & 1.20 & 0.950 & 0.902 & 6.4 & 0.64 & 1.076 \\
0 & 12 & 21 & 1.10 & 0.973 & 0.882 & 6.2 & 0.66 & 1.038 \\
\hline 2 & 12 & 4 & 1.67 & 0.805 & 0.884 & 46 & 0.37 & 1.104 \\
2 & 12 & 7 & 1.33 & 0.866 & 0.817 & 43 & 0.40 & 1.026 \\
2 & 12 & 11 & 1.20 & 0.903 & 0.780 & 41 & 0.41 & 1.003 \\
2 & 12 & 21 & 1.10 & 0.942 & 0.745 & 39 & 0.43 & 0.994 \\
\hline
\end{tabular}
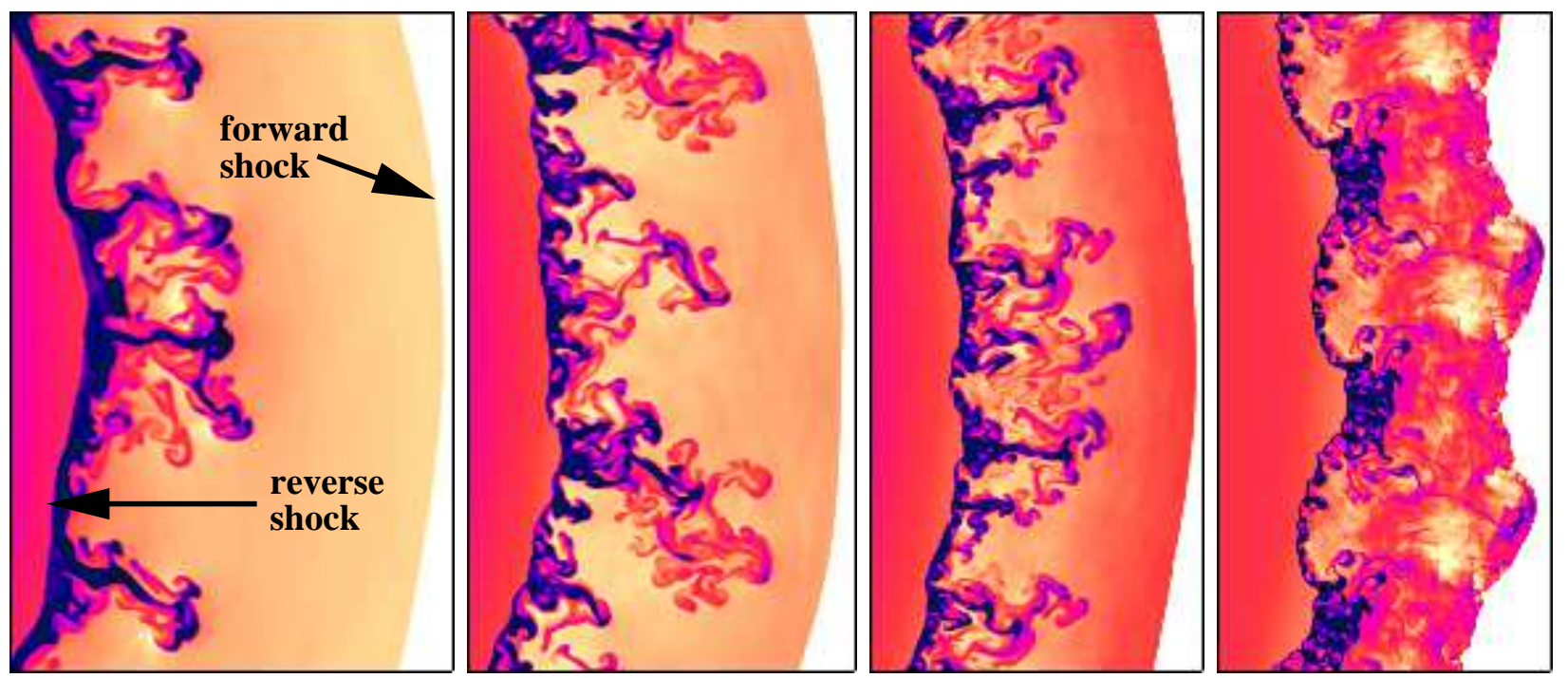

Fig. 4.- Convective instabilities in driven waves with $n=7$ and $s=2$ and values of $\gamma_{\text {eff }}$ as marked. The thin shell of dense (black is high density, white is low density), shocked ejecta is deformed into narrow fingers, characteristic of the R-T instability. 
In all of the cases listed in Table 1, the layer of shocked ejecta was found to be unstable with an evolution similar to that seen in previous studies (i.e., Chevalier et al. 1992; Jun \& Norman 1996a). As an example, we show the density for driven waves with $n=7, s=2$, and different values of $\gamma_{\text {eff }}$ in Figure 4 . The results for other combinations of $n$ and $s$ are similar. These images are taken at the end of the simulations, once the instability has reached saturation. In addition to shrinking the interaction region, a lower value of $\gamma_{\text {eff }}$ produces a narrower, denser shell of shocked ejecta and sharper, denser R-T fingers. Aside from the narrower interaction region, these results for small $\gamma_{\text {eff }}$ are similar to those found by Chevalier \& Blondin (1995) for driven waves with radiative cooling in the shocked ejecta. In this latter case the ejecta behaved as an ideal gas with $\gamma \approx 1$, while the CSM was modeled as an ideal gas with $\gamma=5 / 3$.

We can obtain a rough estimate of the instability growth rate by following the turbulent energy density in the intershock region. Rather than attempt to subtract off the bulk radial velocity to find the contribution to the turbulent velocity, and to avoid confusion in comparing $2 \mathrm{D}$ and $3 \mathrm{D}$ simulations, we consider only a single angular component of the turbulent motion. Furthermore, to remove the effects of radial expansion, deceleration, and shock compression, we normalize this turbulent energy to the bulk kinetic energy density associated with the shock front. We thus define the ratio

$$
\chi=\frac{\int \rho u_{\theta}^{2} d \tau}{\rho_{1} V_{1}^{2} \int d \tau},
$$

where the integration is only over the volume of shocked gas. The evolution of this turbulent energy parameter is shown in Figure 5, from which one can see that while the saturated levels of turbulent energy density differ by about a factor of two, the growth rates are not noticeably affected by changes in $\gamma_{\text {eff }}$, despite the fact that the radial profile from the spherical solution has changed dramatically (e.g., the density gradient has changed sign).

The saturation of the instability, at least in terms of the width of the mixing region, is also relatively unaffected by changes in $\gamma_{\text {eff }}$. In Figure 6 we plot an angle-averaged radial profile of the ejecta mass fraction. In a spherically-symmetric

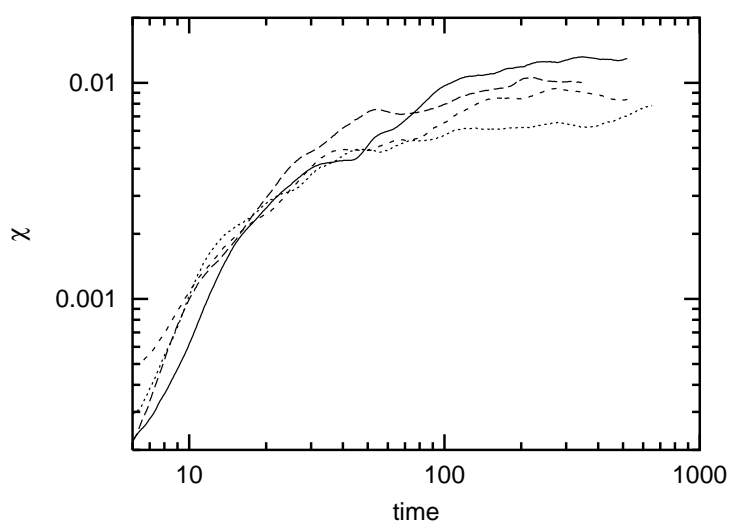

Fig. 5.- Growth of the convective instability as measured by the normalized turbulent energy density, $\chi$, in the interaction region. These curves are from $2 \mathrm{D}$ simulations with $n=7, s=2$, and $\gamma_{\mathrm{eff}}=$ $5 / 3$ (solid), 4/3 (long dash), 1.2 (short dash), and 1.1 (dotted).

model this would be a step function from unity dropping to zero at the contact discontinuity. To provide an easy comparison, we have normalized the radii such that the reverse shock is located at the same radius for all models. From this we can see that the length of the R-T fingers is comparable for all four values of $\gamma_{\text {eff. }}$.

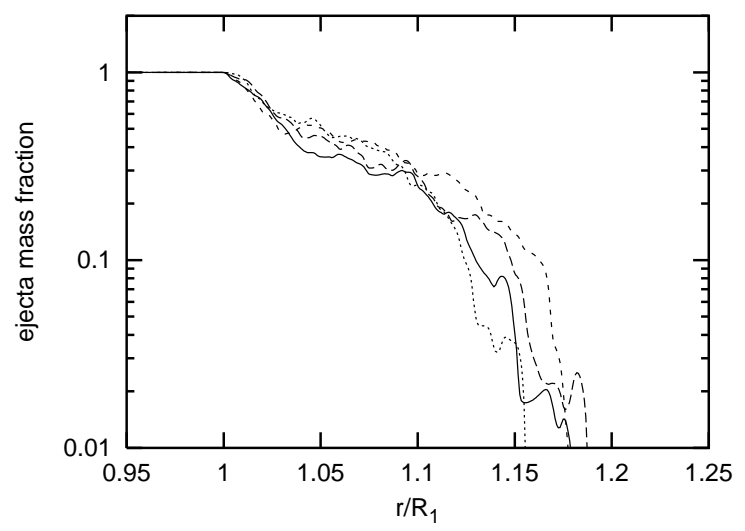

Fig. 6.- The width of the mixing region, as illustrated with the mass fraction of ejecta, is relatively unaffected by changes in $\gamma_{\text {eff }}$. For this comparison we have normalized radii in each model to the reverse shock rather than the forward shock, so we can directly compare the radial extent of the mixing region. The line styles are as in Figure 5. 
While the instability itself is insensitive to changes in the compression ratio, the overall width of the interaction region decreases with increasing compression ratio. This has the important effect

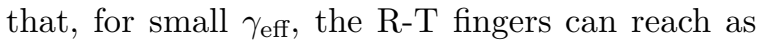
far as the forward shock front. To measure the maximum extent of the R-T fingers, we identify an average mass fraction of 0.001 as the leading edge of the mixing region. Since the radial gradient of the mass fraction is quite steep at these small values, the exact choice of a cutoff value does not affect the results. The time dependence of the maximum radial extent (relative to the forward shock) of the R-T fingers is illustrated in Figure 7. In the run with the highest compression ratio $\left(\gamma_{\text {eff }}=1.1\right)$, the R-T fingers quickly reach and pass the average shock radius. We note that when the curves in Figure 5 become approximately flat, the system is in a near self-similar state and the ratio of the forward shock radius to the contact discontinuity radius remains approximately constant. If we allowed the system to evolve to the point where the reverse shock entered the plateau region in the ejecta density (which nominally happens when the mass of swept up CSM is comparable to the ejecta mass), the ratio of the forward shock radius to the contact discontinuity radius would start to increase and the R-T fingers would drop behind the forward shock.

Once the fingers of dense, shocked ejecta reach the forward shock, they can begin to distort the outer blast wave by pushing small regions out ahead of the average shock radius. The resulting bumps in the outer shock can be seen in Figure 4 for the simulation with $\gamma_{\text {eff }}=1.1$. The shock front is nearly spherical for large values of $\gamma_{\mathrm{eff}}$, while values close to unity produce significant deviations from spherical symmetry.

We note that even in the most dramatic case of $n=12, s=0$, and $\gamma_{\mathrm{eff}}=1.1$, the deviation from a spherical outer shock remains relatively small; the penetrating fingers are not able to dramatically alter the forward shock. This can be seen in Figure 7 , where the maximum radial extent of the fingers appears capped at $\sim R_{1}$. Since the instability is relatively unaffected by the value of $\gamma_{\text {eff }}$, we expect Figure 7 to show a series of parallel curves as the fingers grow until saturation occurs (at several 100 times the initial simulation time, although this depends on the magnitude of the initial perturba-

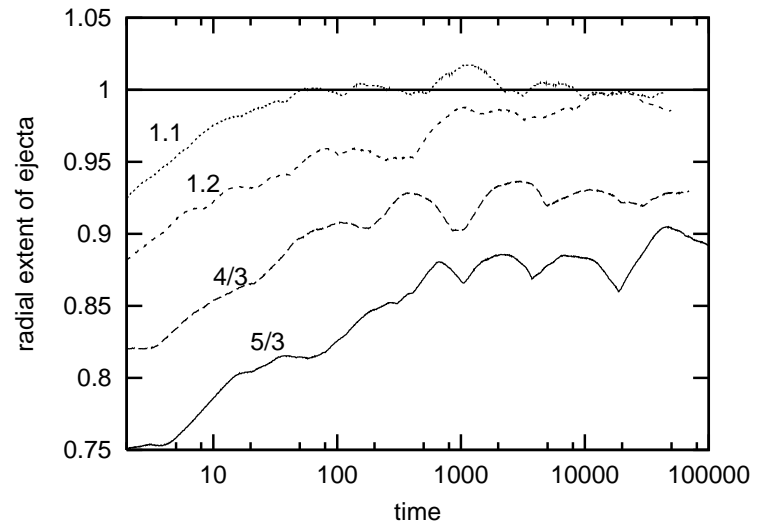

Fig. 7.- The maximum radial extent (measured relative to the average radius of the outer shock) of the R-T fingers of dense shocked ejecta is shown as a function of time for $n=7$ and $s=2$. For the smallest value of $\gamma_{\text {eff }}$, the fingers reach all the way to the shock front. For the other values of $\gamma_{\text {eff }}$ the R-T fingers reach a maximum length after $t \sim 1000$.

tion). If the fingers reach the forward shock before saturation, as in the $\gamma_{\text {eff }}=1.1$ case, they cannot push out much beyond the shock and their growth stalls. This limited ability to affect the shock front is due to the strong shearing flow created when the shock front is distorted. If a clump of dense ejecta has sufficient radial momentum to push out the forward shock in a local protrusion, the deformed shock front generates a substantial tangential post shock flow around the ejecta clump. In all of our simulations, this shear flow quickly disrupts the clump through the Kelvin-Helmholtz instability, with the remnants of the clump quickly advected back into the interaction region. Thus we never see a local protrusion of the shock front stick out more than a few percent of the blast wave radius.

\subsection{D SIMULATIONS}

We repeated two simulations in 3D to check that these results are not dramatically affected by the assumption of axisymmetry, using $n=12$ and $s=0$ for $\gamma_{\mathrm{eff}}=5 / 3$ and 1.1. In comparing the $2 \mathrm{D}$ and $3 \mathrm{D}$ simulations, we stress that the numerical code remained virtually identical. The only changes involved using a 3D grid and repeating the hydrodynamic updates in a third direction. 

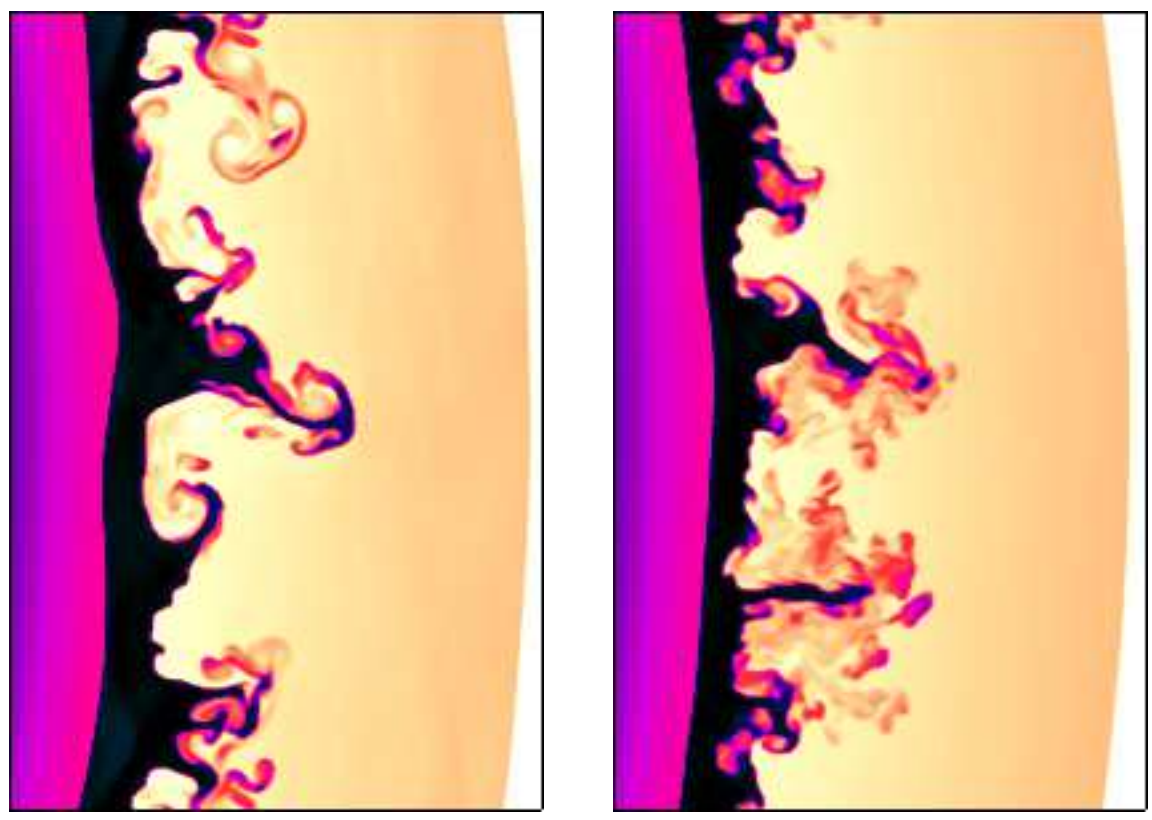

Fig. 8. - A comparison of the shock structure in a $2 \mathrm{D}$ axisymmetric simulation (left), with a planar slice from a 3D simulation (right). The shading depicts gas density, with black representing the high density of the shocked ejecta and white the low density of the unshocked CSM. The location of the forward shock is marked by a solid black line. These runs used $n=12, s=0$, and $\gamma_{\text {eff }}=5 / 3$.
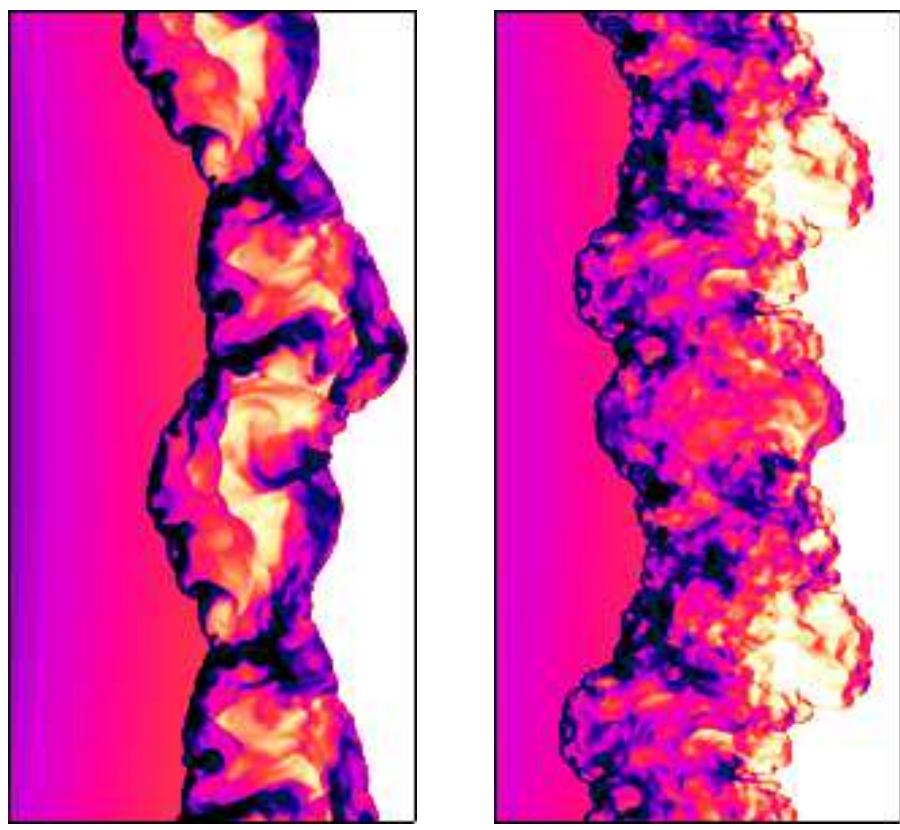

Fig. 9.- A comparison of the shock structure in a 2D axisymmetric simulation (left), with a planar slice from a 3D simulation (right). These runs used $n=12, s=0$, and $\gamma_{\mathrm{eff}}=1.1$. 
For the more familiar case of $\gamma_{\text {eff }}=5 / 3$ our results are quite similar to previous 3D simulations of driven waves (e.g., Jun \& Norman 1996b), showing only minor differences between $2 \mathrm{D}$ and $3 \mathrm{D}$ simulations. This similarity is exhibited in Figure 8. The only significant difference visible in this Figure is the increased amount of small-scale structure in the 3D simulation, although this difference does not appear to affect any of the global properties of the driven wave. A quantitative comparison of these two simulations finds that the growth and saturation of the turbulent energy is virtually identical, the radial extent of the fingers is comparable (the 3D fingers reached slightly further than the 2D fingers), the forward shock remains spherical and the width of the interaction region grows slightly in both cases as the instability approaches saturation.

The situation is somewhat more complicated for the case of high shock compression, as shown in Figure 9. Here one sees much more small-scale structure in the 3D simulation, both within the interaction region and in the forward and reverse shocks. This more dramatic difference between 2D and $3 \mathrm{D}$ in the high-compression case is consistent with the fact that the R-T fingers do not reach the shock front when the shock compression is low, but they do reach - and perturb - the forward shock when the compression is large. For both values of $\gamma_{\text {eff }}$ we see more small-scale structure in the R-T fingers in $3 \mathrm{D}$, but in the high-compression case this small-scale structure can modify the forward shock to produce many small wavelength, but large amplitude, perturbations.

A consequence of the more oblique shocks created by the deformation of the forward shock in the $3 \mathrm{D}$ simulation is an overall drop in the average compression ratio and a corresponding increase in the width of the interaction region. Despite these differences, our statistical measures of the convective instability remain relatively unaffected by the dimensionality of the simulation. Furthermore, our primary conclusion from the $2 \mathrm{D}$ simulations still holds in 3D; the R-T fingers are able to deform the forward shock when the compression ratio is high.

\section{DISCUSSION}

If young supernova remnant shocks accelerate cosmic rays with high efficiency, the acceleration process can cause these high Mach number shocks to have compression ratios considerably greater than four. We have investigated the effects of this high compression on the hydrodynamic stability of young remnants with a simple and direct method; we perform hydrodynamic simulations with the effective ratio of specific heats, $\gamma_{\text {eff }}$, set to values less than $5 / 3$, yielding compression ratios, $\sigma \simeq\left(\gamma_{\text {eff }}+1\right) /\left(\gamma_{\text {eff }}-1\right)>4$. While this fluid approach is clearly an approximation to the real situation where the collisionless shocks accelerate particles to relativistic energies, we believe it accounts for the major effects of efficient particle acceleration on the overall dynamics and, most importantly, on the Rayleigh-Taylor instabilities that develop in the interaction region between the forward and reverse shocks.

Somewhat surprisingly, we find that a large compression ratio has little effect on the growth of R-T instabilities. However, since a large $\sigma$ dramatically shrinks the width of the interaction region between the forward and reverse shocks, several important effects emerge from of our highcompression simulations:

(1) In contrast to shocks with $\sigma \sim 4$, R-T fingers reach closer to the forward shock front, and if $\sigma$ is large enough, ejecta material can be found at, and even slightly ahead of the average shock radius. This can occur fairly quickly after the explosion (depending on how quickly a reverse shock forms and the magnitude of any inhomogeneities seeding this instability) and the fingers should stay near the forward shock front throughout the time the reverse shock is in the power law portion of the ejecta density profile. In general this should apply to SNRs from an age of only months up to several 1000 years.

(2) The forward shock is perturbed on short wavelengths and with relatively small amplitudes. In addition to slightly altering the spherical shape of the shock front, this will lead to some small spread in the postshock temperatures. Note also that the overall temperature of the shocked gas will be substantially lower if particle acceleration is efficient and compression ratios are large than if little acceleration occurs (e.g., Ellison et al. 
2000). This effect provides a coupling between the shock morphology and thermal X-ray emission (Decourchelle, Ellison, \& Ballet 2000; Hughes, Rakowski, \& Decourchelle 2000b).

(3) If the mixing region reaches the forward shock, the morphology of the magnetic field as seen through radio synchrotron polarization observations may be affected. Jun \& Norman (1996b) followed the evolution of the ambient magnetic field in driven wave simulations, looking for an explanation for the origin of observed polarization in young SNRs in the elongation of the field by R-T instabilities. Our work offers a ready explanation for why this polarization can extend all the way to the forward shock.

While there are clearly differences between the actual situation in SNRs where some fraction of swept-up material is shock accelerated to relativistic energies, and simply lowering $\gamma_{\text {eff }}$, we believe our results are qualitatively correct. The important differences include the fact that lowering $\gamma_{\text {eff }}$ implies that the effects of relativistic particle pressure and particle loss occur everywhere rather than just in the shocked gas and in the precursor regions in front of the forward and reverse shocks. We note that if the finite size of the shock is limiting the maximum particle energy, there will be a significant fraction of the total pressure in high energy particles with upstream diffusion lengths on the order of $1 / 10$ of the shock radius. This difference in pressure distribution will have some effect on the shock evolution since, instead of a uniform $\gamma_{\text {eff }}$, the actual remnant has a gas with a soft equation of state pushing one with a harder equation of state. While we have not tested this difference in detail, we expect the effects we found will actually be enhanced if $\gamma_{\text {eff }}$ varies spatially.

Another difference comes about because we use eq. (1) with a constant $\gamma_{\text {eff }}$ to determine $\sigma$ instead of eqs. (2). With (1), there is little variation in $\sigma$ as the SNR evolves as long as $M_{\mathrm{S} 0} \gg 1$, while eqs. (2) can give a much larger variation depending on the parameters. ${ }^{3}$ In any case, since in all probability $\sigma \gg 4$ during the time we consider before

\footnotetext{
${ }^{3}$ We note that it is not always the case that lowering the Mach numbers causes $\sigma$ to decrease. Equations (2) apply when injection into the shock acceleration mechanism is efficient. If injection is weak enough, high Mach number, test-particle solutions with $\sigma \simeq 4$ can result (see Berezhko \& Ellison 1999, for a full discussion).
}

the reverse shock enters the ejecta plateau region, any differences resulting from $\sigma$ varying with time are likely to be small and go in the direction of increasing the effects we report.

Finally, since the shrinking of the interaction region between the forward and reverse shocks depends totally on the pressure in that region, effects other than particle acceleration which influence the pressure may be important. The most likely effect which we have neglected comes from the compression of the magnetic field, $B$. Since the magnetic pressure scales as $B^{2}$, increasing the compression ratio could produce magnetic pressures large enough to prevent the interaction region from becoming narrow enough to allow the R-T fingers to reach the forward shock. This, of course, will depend on the Alfvén Mach number and the angle the upstream field makes with the shock. The pressure effects of the magnetic field will be offset somewhat by the fact that, for a given compression ratio, the shocked thermal pressure is considerably less in a shock undergoing efficient acceleration compared to one with a low $\gamma_{\text {eff }}$ and no acceleration.

We thank A. Decourchelle, J. P. Chièze, and S. Reynolds for helpful discussions. The numerical simulations reported here were performed at the North Carolina Supercomputing Center using 100 processors of an IBM SP2. We thank NCSC and IBM for their generous support of computing resources. Support for this work was provided by NASA under grant NAG-7153.

\section{REFERENCES}

Allen, G. E. et al. 1997, ApJ 487, L97

Axford, W. I. 1981, Proc. 17th Int. Cosmic Ray Conf. (Paris), 12, 155

Baring, M. G., Ogilvie, K. W., Ellison, D. C., \& Forsyth, R. J. 1997, ApJ, 476, 889

Bartel, N., Rupen, M. P., Shapiro, I. I., Preston, R. A., \& Rius, A. 1991, Nature, 350, 212

Berezhko, E. G., Ellison, D. C. 1999, ApJ, 526, 385

Blandford, R. D., \& Eichler, D, 1987, Physics Reports, 154,1 
Chevalier, R. A. 1982a, ApJ, 258, 790

Chevalier, R. A. 1982b, ApJ, 259, L85

Chevalier, R. A. 1983, ApJ, 272, 765

Chevalier, R. A., \& Blondin, J. M. 1995, ApJ, 444, 312

Chevalier, R. A., Blondin, J. M., \& Emmering, R. T. 1992, ApJ, 392, 118

Decourchelle, A., Ellison, D. C. \& Ballet, J., 2000, ApJ, 543, L57

Dickel, John R., Eilek, Jean A., Jones, E. M., \& Reynolds, S. P. 1989, ApJS, 70, 497

Dickel, J. R., van Breugel, W. J. M., \& Strom, R. G. 1991, AJ, 101, 2151

Dorfi, E. A. 2000, Ap\&SS, 272, 227

Dorfi, E. A., \& Böhringer, H. 1993, A\&A, 273, 251

Drury, L.O'C., \& Falle, S. A. E. G. 1986, MNRAS, 223,353

Drury, L.O'C., Markiewicz, W.J., \& Völk, H.J. 1989, A\&A, 225, 179

Dwarkadas, V. V., \& Chevalier, R. A. 1998, ApJ, 497, 807

Eichler, D. 1981, ApJ, 247, 1089

Ellison, D. C., Berezhko, E. G., \& Baring, M. G. 2000, ApJ, 540, 292

Ellison, D. C., \& Eichler, D. 1984, ApJ, 286, 691

Ellison, D.C., Möbius, E., \& Paschmann, G. 1990, ApJ, 352, 376

Fesen, R. A., \& Gunderson, K. S. 1996, ApJ, 470, 967

Gull, S. F. 1973, MNRAS, 161, 47

Hughes, J. P., Rakowski, C. E., Burrows, D. N., \& Slane, P. O. 2000a, ApJ, 528, L109

Hughes, J. P., Rakowski, C. E. \& Decourchelle, A. 2000b, 543, L61

Jun, B.-I., \& Norman, M. L. 1996a, ApJ, 465, 800

Jun, B.-I., \& Norman, M. L. 1996b, ApJ, 472, 245
Jun, B.-I., Jones, T. W., \& Norman, M. L. 1996, ApJ, 468, L59

Kang, H. \& Jones, T. W. 1997, ApJ, 476, 875

Kazanas, D., \& Ellison, D. C. 1986, ApJ, 304, 178

Koyama, K., Petre, R., Gotthelf, E. V., Hwang, U., Matsura, M., Ozaki, M., Holt, S. S. 1995, Nature, 378, 255

Malkov, M. 1997, ApJ, 485, 638

Mastichiadis, A. \& de Jager, O. C. 1996, A\&A, 311, L5

McClements, K. G., Dendy, R. O., Drury, L. O'C., \& Duffy, P. 1996, MNRAS, 280, 219

Reynolds, S. P. and Keohane, J.W., 1999, ApJ, 525,368

Scholer, M., Trattner, K.J., \& Kucharek, H. 1992, ApJ, 395, 675

Shirkey, R. C. 1978, ApJ, 224, 477

Tanimori, T., et al. 1998, ApJ, 497, L25

Terasawa, T., et al. 1999, Proc. 26th Int. Cosmic Ray Conf. (Salt Lake City), 6, 528

Wang, C.-Y. \& Chevalier, R. A. 2000, ApJ, 549, 1119

This 2-column preprint was prepared with the AAS LATEX macros v5.0. 